\title{
Synergistic Block of SARS-CoV-2 Infection by Combined Drug Inhibition of the Host Entry Factors PIKfyve Kinase and TMPRSS2 Protease
}

\author{
(D) Alex J. B. Kreutzberger, a,b Anwesha Sanyal, a,b (D) Ravi Ojha,c (D) Jesse D. Pyle,d (D) Olli Vapalahti, ${ }^{c, e, f}$ \\ (D) Giuseppe Balistreri,, ,e (D)Tom Kirchhausen ${ }^{a, b, g}$ \\ aDepartment of Cell Biology, Harvard Medical School, Boston, Massachusetts, USA \\ bProgram in Cellular and Molecular Medicine, Boston Children's Hospital, Boston, Massachusetts, USA \\ cDepartment of Virology, Faculty of Medicine, University of Helsinki, Helsinki, Finland \\ aprogram in Virology, Harvard Medical School, Boston, Massachusetts, USA \\ eDepartment of Veterinary Biosciences, University of Helsinki, Helsinki, Finland \\ Virology and Immunology, Helsinki University Hospital Diagnostic Center (HUSLAB), Helsinki, Finland \\ sDepartment of Pediatrics, Harvard Medical School, Boston, Massachusetts, USA
}

ABSTRACT Repurposing FDA-approved inhibitors able to prevent infection by severe acute respiratory syndrome coronavirus 2 (SARS-CoV-2) could provide a rapid path to establish new therapeutic options to mitigate the effects of coronavirus disease 2019 (COVID-19). Proteolytic cleavages of the spike (S) protein of SARS-CoV-2, mediated by the host cell proteases cathepsin and TMPRSS2, alone or in combination, are key early activation steps required for efficient infection. The PIKfyve kinase inhibitor apilimod interferes with late endosomal viral traffic and through an ill-defined mechanism prevents in vitro infection through late endosomes mediated by cathepsin. Similarly, inhibition of TMPRSS2 protease activity by camostat mesylate or nafamostat mesylate prevents infection mediated by the TMPRSS2-dependent and cathepsin-independent pathway. Here, we combined the use of apilimod with camostat mesylate or nafamostat mesylate and found an unexpected $\sim 5$ - to 10-fold increase in their effectiveness to prevent SARS-CoV-2 infection in different cell types. Comparable synergism was observed using both a chimeric vesicular stomatitis virus (VSV) containing S of SARSCoV-2 (VSV-SARS-CoV-2) and SARS-CoV-2. The substantial $\sim 5$-fold or higher decrease of the half-maximal effective concentrations $\left(\mathrm{EC}_{50} \mathrm{~s}\right)$ suggests a plausible treatment strategy based on the combined use of these inhibitors.

IMPORTANCE Infection by severe acute respiratory syndrome coronavirus 2 (SARSCoV-2) is causing the coronavirus disease 2019 (COVID-2019) global pandemic. There are ongoing efforts to uncover effective antiviral agents that could mitigate the severity of the disease by controlling the ensuing viral replication. Promising candidates include small molecules that inhibit the enzymatic activities of host proteins, thus preventing SARS-CoV-2 entry and infection. They include apilimod, an inhibitor of PIKfyve kinase, and camostat mesylate and nafamostat mesylate, inhibitors of TMPRSS2 protease. Our research is significant for having uncovered an unexpected synergism in the effective inhibitory activity of apilimod used together with camostat mesylate or nafamostat mesylate.

KEYWORDS COVID-19, SARS-CoV-2, synergism, virus entry

evere acute respiratory syndrome coronavirus 2 (SARS-CoV-2) infection has caused the global pandemic known as coronavirus disease 2019 (COVID-2019). Currently, there is no widespread use of an antiviral agent against the disease, but several
Citation Kreutzberger AJB, Sanyal A, Ojha R Pyle JD, Vapalahti O, Balistreri G, Kirchhausen T. 2021. Synergistic block of SARS-CoV-2 infection by combined drug inhibition of the host entry factors PIKfyve kinase and TMPRSS2 protease. J Virol 95:e00975-21. https://doi.org/10.1128/JVI .00975-21.

Editor Tom Gallagher, Loyola University Chicago

Copyright $\odot 2021$ American Society for Microbiology. All Rights Reserved. Address correspondence to Tom Kirchhausen, kirchhausen@crystal.harvard.edu.

Received 15 June 2021 Accepted 12 August 2021

Accepted manuscript posted online 18 August 2021

Published 13 October 202 
candidates have been identified (1-6). Apilimod is currently in a clinical trial for the prevention of SARS-CoV-2 infections in the United States (ClinicalTrails.gov identifier NCT04446377). The TMPRSS2 protease inhibitor camostat mesylate has been tested with hospitalized COVID-19 patients in Denmark (7) and is in a clinical trial on adult COVID-19 patients in France (ClinicalTrails.gov identifier NCT04608266), while the higher-affinity TMPRSS2 protease inhibitor nafamostat mesylate is being used in COVID-19-related trials in Russia (ClinicalTrials.gov identifier NCT04623021), South Korea (ClinicalTrails.gov identifier NCT04418128), and Japan (8).

Targeting the entry route of SARS-CoV-2 has been particularly challenging because there appear to be at least two different pathways for virus entry into cells (1). SARSCoV-2 entry is mediated by the virus spike $(S)$ protein (9-11) and requires the receptor ACE2 in the host cell $(1,12,13)$. Upon engagement of the ACE2 receptor, the spike protein catalyzes the fusion of the viral membrane envelope with a host cell membrane to release the contents of the virus into the cytoplasm of host cells. For the spike protein to facilitate this reaction, it must first be cleaved by a host cell protease $(1,10)$. This can be accomplished by different host proteases, including TMPRSS2 and TMPRSS4 (14), factor Xa $(15,16)$, and cathepsins during endocytosis (1). The protease inhibitors E-64 and camostat mesylate target cathepsin and TMPRSS2 and inhibit SARS-CoV-2 infection (1), but their low (micromolar) affinities have made them unlikely candidates for clinical use. Nafamostat mesylate inhibits TMPRSS2 with a high (nanomolar) affinity $(2,6)$. Apilimod has been shown to function in the endosomal pathway by inhibiting PIKfyve kinase, causing a defect in viral trafficking prior to entry (3), but how this relates to the cathepsin or TMPRSS2 protease-dependent pathways has not been investigated.

Using a chimeric vesicular stomatitis virus (VSV) in which the attachment and fusion glycoprotein G is replaced by the S protein of SARS-CoV-2 (VSV-SARS-CoV-2) (17), we investigate inhibition of infection in different cell types known to contain different levels of cathepsin and TMPRSS2 proteases. We tested combinations of the protease inhibitors E-64 (cathepsin), camostat mesylate, and nafamostat mesylate (TMPRSS2) and the lipid kinase inhibitor apilimod (PIKfyve kinase) on VSV-SARS-CoV-2 infection of multiple cell types. We observed a 5 -fold synergistic effect on infection by VSV-SARSCoV-2 by the simultaneous inhibition of TMPRSS2 and PIKfyve kinase. Furthermore, the synergistic inhibitory effects on infection by VSV-SARS-CoV-2 or a clinical isolate of SARS-CoV-2 were observed with nanomolar concentrations of camostat mesylate and apilimod. This finding suggests that a combination of inhibitors that target two different host factors in the entry pathway of SARS-CoV-2 will likely be more effective than targeting either one alone.

\section{RESULTS AND DISCUSSION}

VSV-SARS-CoV-2 infection is partially prevented by PIKfyve kinase or TMPRSS2 protease inhibitors. It has been proposed that two distinct host proteases, TMPRSS2 and cathepsin, facilitate different SARS-CoV-2 viral entry routes, cell surface or endosomal, and their abundance in different cell types may influence the entry pathway (1). To test this, we employed a panel of inhibitors on SARS-CoV-2 infection in African green monkey kidney epithelium-derived VeroE6 cells poorly expressing TMPRSS2, VeroE6 cells stably expressing ectopic TMPRSS2, as well as human colon carcinoma-derived Caco- 2 cells and human lung-derived Calu-3 cells naturally expressing TMPRSS2.

We first established the relative importance of the cathepsin-dependent route for infection by VSV-SARS-CoV-2 by determining the effect of the cathepsin inhibitor E-64 on the expression of enhanced green fluorescent protein (eGFP) mediated by VSVSARS-CoV-2. As summarized in the plots in Fig. 1A, we found a cell type dependence of the extent of the infection block with a half-maximal effective concentration $\left(E C_{50}\right)$ in the $\sim 5$ to $10 \mu \mathrm{M}$ range. Notably, only VeroE6 cells treated with E-64 displayed a full infection block, the result expected for cells expressing cathepsin but no TMPRSS2; in contrast, cells expressing cathepsin and TMPRSS2 (VeroE6+TMPRRS2 and Caco-2) displayed a partial block, while Calu-3 cells with minimal expression of cathepsin $L$ (18) did not respond, as expected, to E-64 treatment (1). 
A

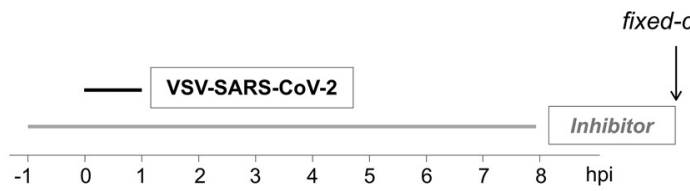

B VSV-SARS-CoV-2
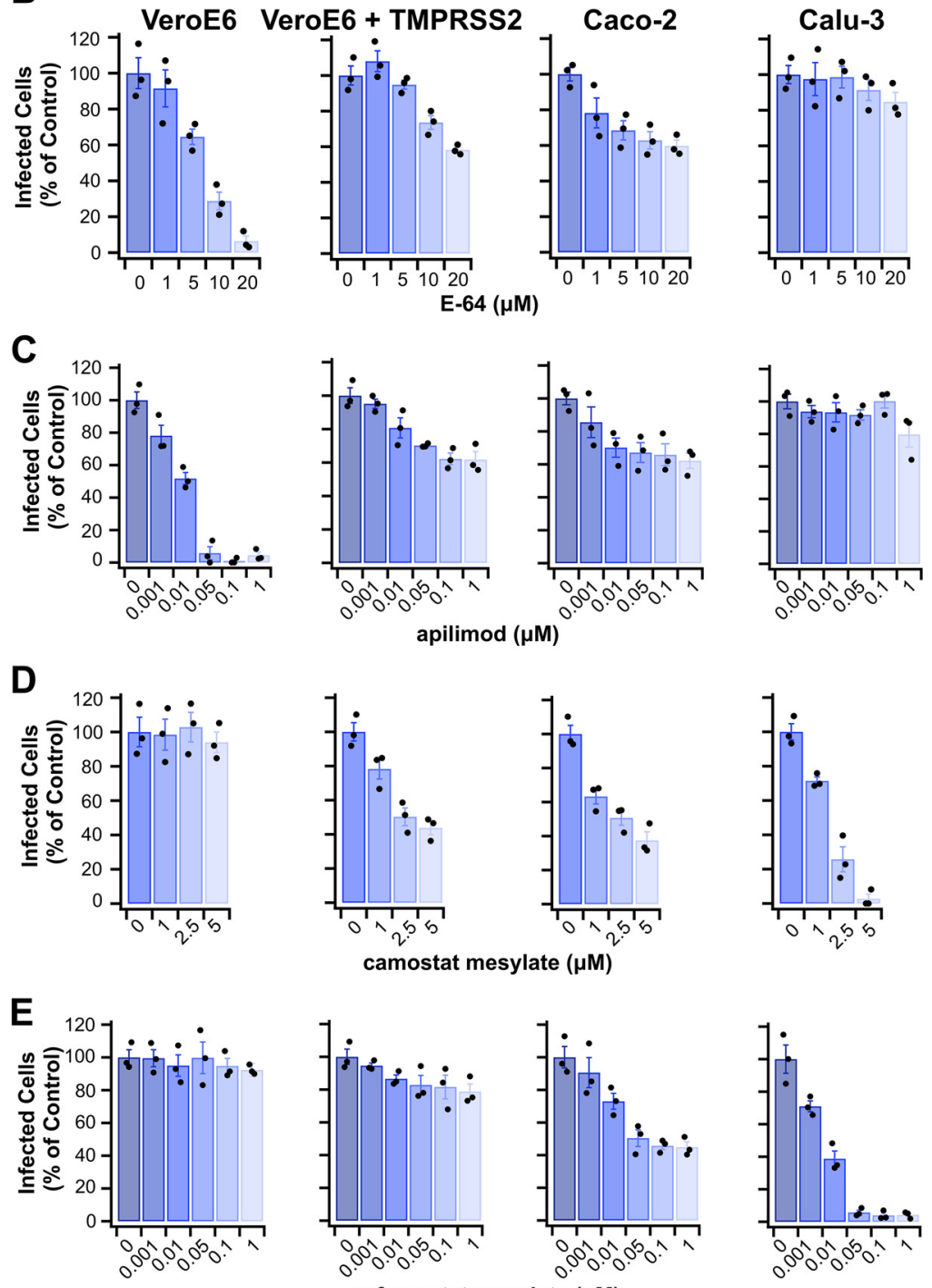

$\mathbf{F}$ nafamostat mesylate $(\mu \mathrm{M})$

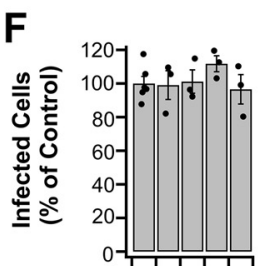

VSV
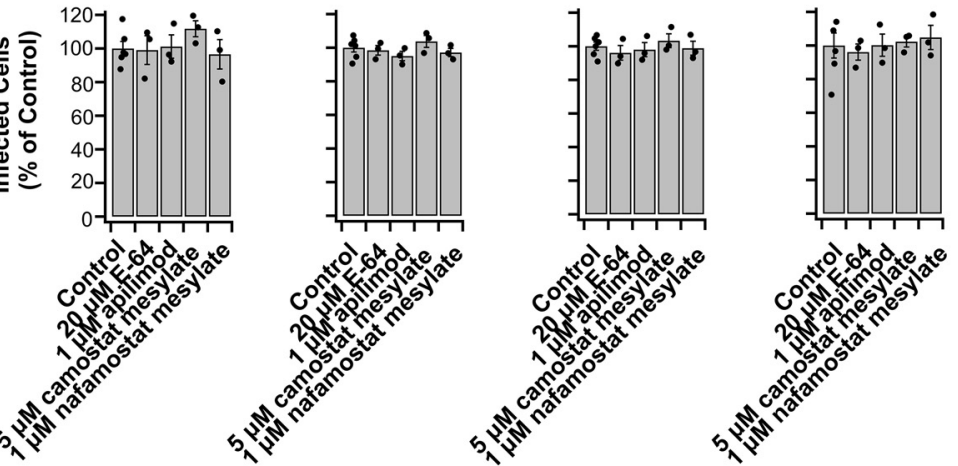

FIG 1 The protease inhibitors E-64, apilimod, camostat mesylate, and nafamostat mesylate prevent infection by VSV-SARS-CoV-2 but not by VSV. (A) Schematic of the infectivity assay for cells pretreated

(Continued on next page) 
We extended the analysis and studied the inhibitory effect of the PIKfyve kinase inhibitor apilimod on VSV-SARS-CoV-2 infection (Fig. 1B). We found that all cell types shown to be sensitive to E-64 also responded to treatment with apilimod with an $\mathrm{EC}_{50}$ of $\sim 10 \mathrm{nM}$, whereas the Calu-3 cell line, which is insensitive to E-64 (19), did not respond. Although apilimod does not inhibit cathepsin B or $L(20)$, these observations are consistent with the potential modulation of the endosomal availability of cathepsin by the activity of PIKfyve kinase (21, 22).

A similar analysis, to document the reduction of viral infection using camostat mesylate to block the protease activity of TPMRSS2 (Fig. 1C) or nafamostat mesylate (Fig. 1D), showed, as anticipated, no response in VeroE6 cells lacking TMPRSS2, partial inhibition in VeroE6 or Caco-2 cells expressing TMPRSS2, or full inhibition in Calu-3 cells naturally expressing TMPRSS2 but insensitive to cathepsin inhibition.

These observations are consistent with previous results $(1,2,6,19)$ that infection by VSV-SARS-CoV-2 occurred through two complementary entry pathways with different importances depending on the cell type, one depending on the proteolytic activity of cathepsin and the second relying on the proteolytic activity of TMPRSS2. As a negative control for these experiments, we included infection by the parental VSV (Fig. 1E), whose ability to infect host cells is known to be independent of the enzymatic activities of cathepsin, TMPRSS2, or PIKfyve kinase $(1-4,6)$. As expected, none of the inhibitors for these enzymes influenced the extent of VSV infectivity in any of the cells used. From these results, we could also exclude potential cytotoxic effects of the compounds in the concentration range used.

Synergistic prevention of VSV-SARS-CoV-2 infection by the combined use of PIKfyve kinase and TMPRSS2 protease inhibitors. Since the cathepsin- and TMPRSS2dependent activation of SARS-CoV-2 corresponds to complementary entry pathways thought to act independent of each other, we expected an additive inhibitory effect upon their simultaneous inhibition in cells that express both proteases. Indeed, VSV-SARS-CoV-2 infection of VeroE6 cells expressing cathepsin and TMPRSS2 (VeroE6+TMPRSS2) were equally inhibited ( $\mathrm{EC}_{50}$ of $\sim 2 \mu \mathrm{M}$ ) by camostat mesylate in the absence or presence of increasing concentrations of E-64 up to $20 \mu \mathrm{M}$, the concentration at which E-64 maximally blocked VSV-SARS-CoV-2 infection (Fig. 2A). We used SynergyFinder 2.0 (23) to compare the combined response obtained experimentally with the expected outcome calculated by the Bliss synergy-scoring model. Using this reference model, which considers the multiplicative effect of single drugs as if they acted independently $(23,24)$, we obtained an overall $\delta$-score of 6 , indicative of an additive interaction between camostat and E-64. At combined high levels of camostat and E-64, these protease inhibitors also showed weak concentration dependence, consistent with the prediction of a published model (25). In contrast, the combined use of camostat mesylate and apilimod led to enhanced inhibition of VSV-SARS-CoV-2 infection, with an $\sim 2$-fold decrease in the $\mathrm{EC}_{50}$ of camostat mesylate (from 1 to $0.4 \mu \mathrm{M}$ ) (Fig. 2B), suggestive of a synergistic effect.

We carried out a similar set of experiments using Calu-3 cells, which are known to be deficient in cathepsin $L(18,26)$ and are poorly susceptible to infection by filoviruses mediated by the cathepsin-dependent infection route (18). These cells are insensitive to inhibition of VSV-SARS-CoV-2 and SARS-CoV-2 infection by E-64 $(1,19,27,28)$ (Fig. 1B) and apilimod (19, 29) (Fig. 1C). While the presence of E-64 did not affect the inhibition profile of camostat mesylate (Fig. 2 C), we detected an $\sim 5$-fold decrease from

FIG 1 Legend (Continued)

for $1 \mathrm{~h}$ or not with the inhibitors and subsequently infected with VSV-SARS-CoV-2 for $1 \mathrm{~h}$ in the presence or absence of inhibitors. The cells were incubated for another $7 \mathrm{~h}$ in the presence or absence of inhibitors and then fixed; the percentage of cells expressing eGFP was measured by spinning-disk confocal microscopy. (B to F) Quantification of the number of infected cells from three independent experiments, each determined from 5 fields of view containing 80 to 200 cells per experiment (error bars show standard errors of the means [SEM]) for the indicated cell types. (B and C) Infected Vero (B) or Vero+TMPRSS2 (C) cells were analyzed at $8 \mathrm{hpi}$ using $0.5 \mu \mathrm{g} / \mathrm{ml}$ VSV-SARSCoV-2 RNA. (D and E) Infected Caco-2 (D) or Calu-3 (E) cells were analyzed at 8 hpi using $5 \mu \mathrm{g} / \mathrm{ml}$ VSV-SARS-CoV-2 RNA. (F) Cells infected with $0.075 \mu \mathrm{g} / \mathrm{ml}$ VSV RNA were analyzed at $6 \mathrm{hpi}$. In each case, these virus concentrations and conditions of infection corresponded to an $\mathrm{MOI}$ of $\sim 0.5$. 
A

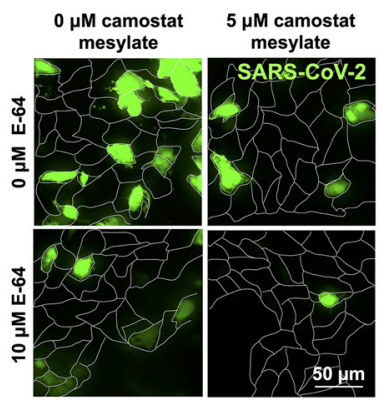

VSV-SARS-CoV-2 : VeroE6 + TMPRSS2

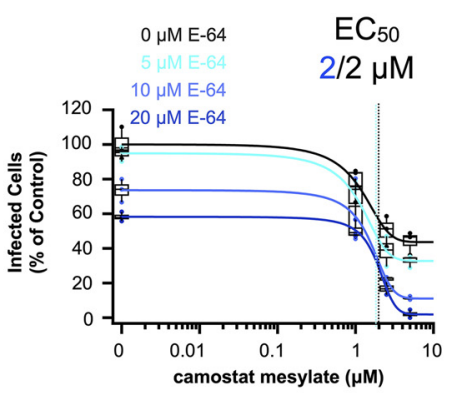

C

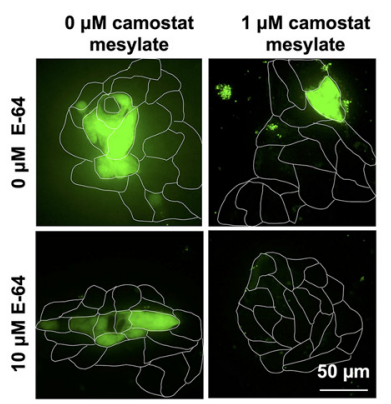

VSV-SARS-CoV-2 : Calu-3

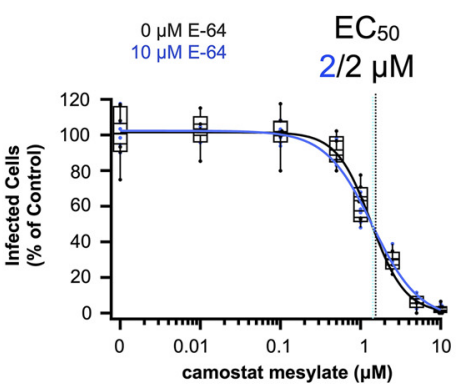

B

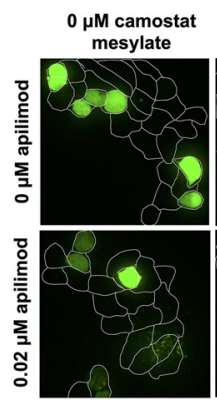

D

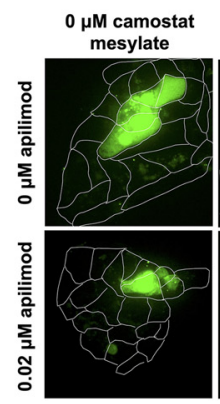

VSV-SARS-CoV-2 : VeroE6 + TMPRSS2

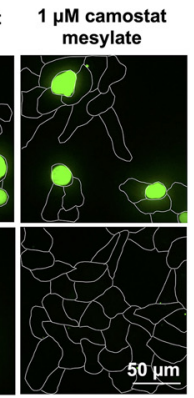

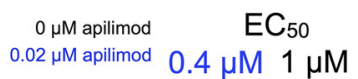

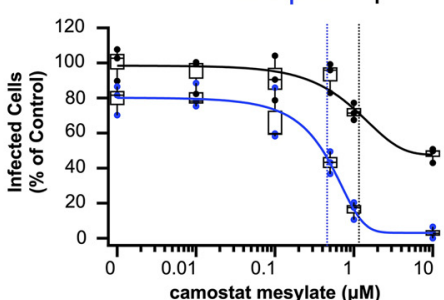

VSV-SARS-CoV-2 : Calu-3

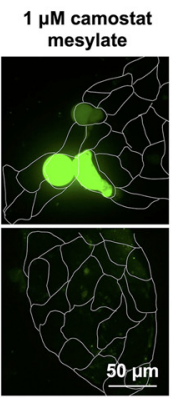

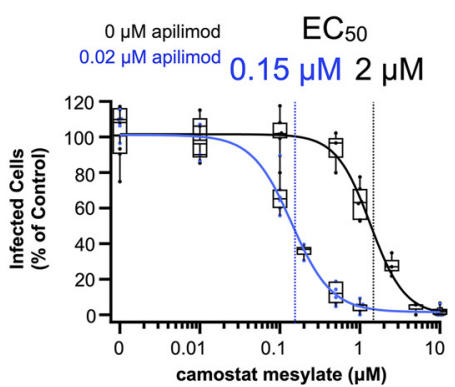

FIG 2 Synergistic inhibition of VSV-SARS-CoV-2 infection by the combined use of apilimod and camostat mesylate. Data are from infection results using VSV-SARS-CoV-2 obtained with different cell types in the absence or the combined presence of increasing concentrations of E-64 and camostat mesylate (A and $C$ ) or apilimod and camostat mesylate (B and D). Representative maximum-Z-projection views (left) are from whole-cell-volume images obtained with optical sections separated by $0.5 \mu \mathrm{m}$ using a spinning-disk confocal microscope; cells were infected with $0.5 \mu \mathrm{g} / \mathrm{ml}$ viral RNA of VSV-SARS-CoV-2 and imaged at $8 \mathrm{hpi}$. Corresponding quantifications of infection (right) are shown in the plots. Each point corresponds to one independent experiment; the

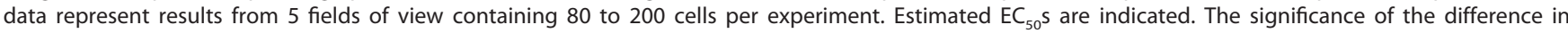
the $\mathrm{EC}_{50} \mathrm{~s}$ was determined by fitting data from replicated experiments and using an unpaired $t$ test. $P$ values were 0.02 for panel $\mathrm{B}$ and 0.002 for panel $\mathrm{D}$; there was no statistically significant difference in the $E C_{50}$ values for the experiments reported in panel $A$ or $C$.

$2 \mu \mathrm{M}$ to $0.2 \mu \mathrm{M}$ in the $\mathrm{EC}_{50}$ of camostat mesylate in cells simultaneously treated with $0.02 \mu \mathrm{M}$ apilimod (Fig. 2D). This result was unexpected given that infection of Calu-3 cells by SARS-CoV-2 did not appear to be affected by apilimod (29) (Fig. 1C).

We extended the synergy analysis to verify the effects upon the simultaneous use of various amounts of apilimod and nafamostat mesylate, another inhibitor of TMPRSS2. Our data also indicate enhanced infection inhibition for nafamostat mesylate, with an $\sim 20$-fold decrease of the $\mathrm{EC}_{50}$ from 0.02 to $0.001 \mu \mathrm{M}$ (Fig. 3A, middle). Simultaneous treatment with apilimod and increasing amounts of nafamostat mesylate led to an analogous enhanced potency of apilimod inhibition, with an $\sim 10$-fold decrease of the $\mathrm{EC}_{50}$ from 0.01 to $0.001 \mu \mathrm{M}$ (Fig. 3A, right). Formal evaluation for synergism using the Bliss reference model was consistent with a synergy $\delta$-score of 41 (a score of $>10$ indicates synergy [23]).

Comparable strong synergistic effects ( $\delta$-score of 34 ) of the combined use of apilimod and nafamostat mesylate were observed in VeroE6 cells expressing TMPRSS2 (Fig. $3 B$, middle and right) infected with VSV-SARS-CoV-2 D614G, whose spike (S) protein includes a point mutation known to increase the infectivity of the native SARS-CoV-2 (30). While this mutation has no effect on the virus entry mechanism or sensitivity to proteases, it stabilizes the stalk region of the spike, which otherwise tends to fall apart after furin cleavage between S1 and S2 (30-33).

Taken together, these synergy results highlight the unexpected nonadditive involvement of PIKfyve kinase activity in the functional effectiveness of TMPRSS2 to mediate viral entry along the TMPRSS2 route.

Synergistic prevention of SARS-CoV-2 infection by the combined use of PIKfyve kinase and TMPRSS2 protease inhibitors. To determine whether SARS-CoV-2 also displays an enhanced block of infection upon the combined use of apilimod and camostat 
A

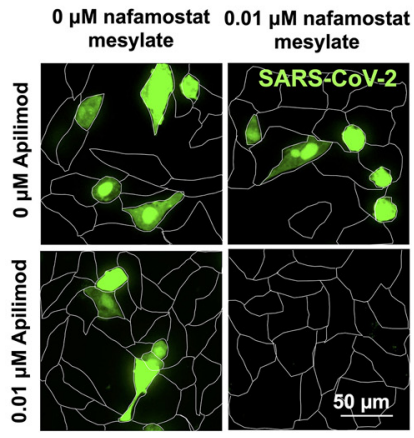

B

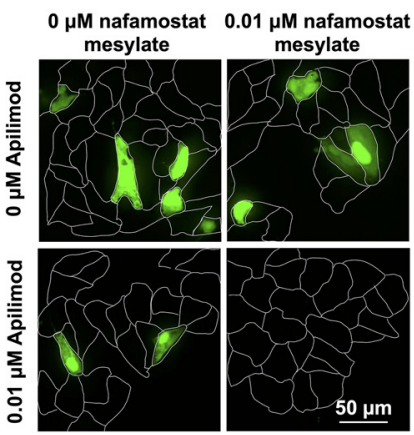

VSV-SARS-CoV-2 : VeroE6 + TMPRSS2
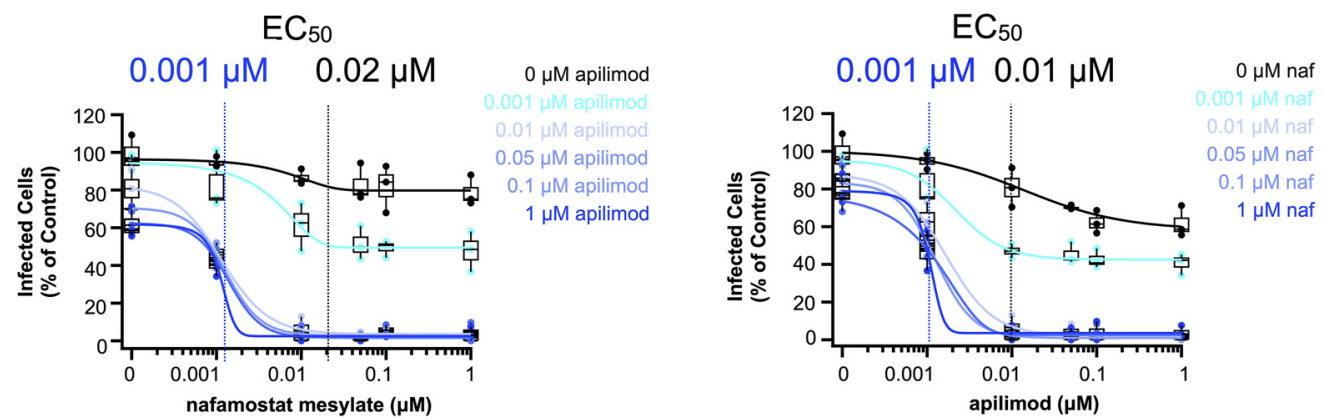

VSV-SARS-CoV-2 D614G : VeroE6 + TMPRSS2

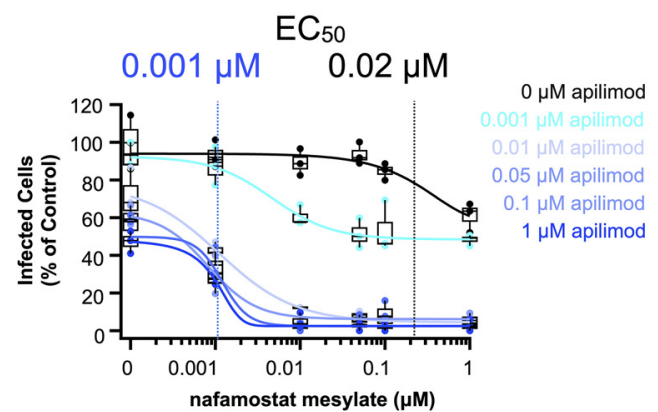

FIG 3 Synergistic inhibition of VSV-SARS-CoV-2 infection by the combined use of apilimod and nafamostat mesylate. Data are from infection results obtained with VeroE6+TPRSS2 cells in the absence or the combined presence of increasing concentrations of apilimod and nafamostat mesylate. Representative maximum-Z-projection views (left) are from whole-cell-volume images obtained with optical sections separated by $0.5 \mu \mathrm{m}$ using a spinningdisk confocal microscope; cells were infected with $0.5 \mu \mathrm{g} / \mathrm{ml}$ viral RNA of VSV-SARS-CoV-2 (A) or $0.2 \mu \mathrm{g} / \mathrm{ml}$ VSV-SARS-CoV-2 D614G viral RNA (B) and imaged at $8 \mathrm{hpi}$. Corresponding quantifications of infection (right) are shown in the plots. Each point corresponds to data from one independent experiment; the data represent results from 5 fields of view containing 80 to 200 cells per experiment. Estimated $\mathrm{EC}_{50} \mathrm{~s}$ are indicated. Plots in both the middle and right panels used the same data.

mesylate, we infected Caco-2 cells ectopically expressing human ACE2 (hACE2), the main receptor for SARS-CoV-2. This cell line was created as a way to enhance the susceptibility of Caco-2 cells to infection by SARS-CoV-2 since the parental cells express low endogenous levels of ACE2 (34); successful infection was scored at $18 \mathrm{~h}$ postinfection (hpi) by the appearance of viral $\mathrm{N}$ protein using immunofluorescence microscopy (Fig. 4, left). The inhibitory $\mathrm{EC}_{50}$ of camostat mesylate decreased $\sim 6$-fold from 0.6 to $0.1 \mu \mathrm{M}$ when used together with apilimod (Fig. 4, right). This observation extends our results from VSV-SARS-CoV-2 chimeras to SARS-CoV-2 and illustrates that the combined chemical inhibition of PIKfyve kinase and TMPRSS2 protease activities is likely to also prevent SARSCoV-2 infection with strong synergy.

Final remarks. According to our present understanding of the early steps necessary for successful infection, SARS-CoV-2 would enter cells using two main redundant routes, each requiring cleavage of the viral S protein: one dependent on the proteolytic activities of members of the cathepsin family and the other requiring proteolysis by TMPRSS2 or similar transmembrane-serine proteases. The expression level of these proteases depends on the cell type, and hence, their relative importance to support successful infection would hinge in part on their relative expression. Cathepsin is primarily found in late endosomes or lysosomes and requires low pH for optimal enzymatic activity; for these reasons, it has been proposed that SARS-CoV-2 entry occurs from the endolysosomal compartment $(35,36)$. In contrast, TMPRSS2 is thought to be located at the cell surface (37), and its optimal proteolytic activity is $\mathrm{pH}$ independent (38); hence, it has been inferred that TMPRSS2 cleavage of $\mathrm{S}$ protein occurs on virions at the plasma membrane, from which viral entry is then assumed to occur (1).

Not surprisingly for protease inhibitors with different targets, the combined inhibition of the enzymatic activities of cathepsin and TMPRSS2 by the respective protease 


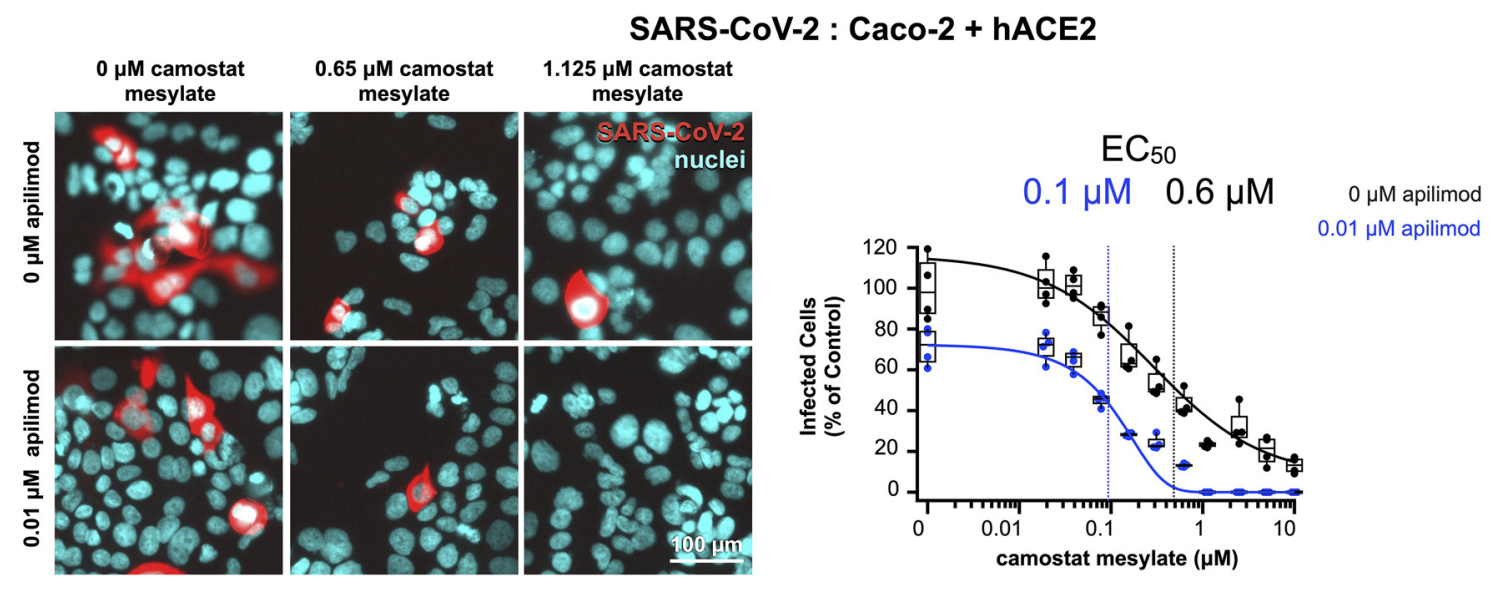

FIG 4 Synergistic inhibition of SARS-CoV-2 infection by the combined use of apilimod and camostat mesylate. Natural SARS-CoV-2 infection inhibition in Caco-2+hACE2 cells ( $\mathrm{MOI}=0.5)$ by camostat mesylate in the absence or presence of apilimod is depicted. Representative examples of images of fixed samples stained with an antibody specific for $\mathrm{N}$ (red) and with Hoechst DNA stain (cyan) to identify the nuclei obtained using wide-field epifluorescence microscopy (left) and corresponding quantifications of infection (right) are shown. Each data point was from 4 independent experiments, representing results from 6,000 to 10,000 cells per experiment. Estimated $E_{50} \mathrm{~s}$ are shown. The significance of the difference $(P$ value of 0.01$)$ in $E C_{50} s$ was determined using an unpaired $t$ test after least-squares nonlinear fitting of the data curves from replicated experiments.

inhibitors E-64 and camostat mesylate led to additive prevention of infection by SARSCoV-2 (1) and the VSV-SARS-CoV-2 chimera (this study).

Apilimod specifically inhibits PIKfyve kinase, thereby blocking the accumulation of phosphatidylinositol 3,5-bisphosphate [PI(3,5)P2], a key phosphoinositide required to modulate the function of a number of proteins involved in late endosomal traffic (3, $21,39,40$ ). While it is now well established that interference with PIKfyve kinase prevents infection by a select group of viruses, including Ebola virus (20), VSV-Ebola virus (3), Marburg virus (20), VSV-SARS-CoV-2 (3), and native SARS-CoV-2 (3-5), the molecular mechanism events responsible for the interference remain to be determined. It has been shown that apilimod has anti-Ebola virus infection synergy with clomiphene citrate ( $\delta$-score of 23.8 [recalculated from data in Fig. 1B in reference 41 using the Bliss reference model $\{23\}])$ and also with other drugs that interfere, by unknown mechanisms, with Ebola virus entry in late endosomes (41). We have now shown synergistic inhibition of SARS-CoV-2 infection by the combined use of apilimod and camostat or apilimod and nafamostat mesylate, even in cells as diverse as Vero cells (which have an active cathepsin entry pathway) and Calu- 3 cells (which lack it).

Prevailing models for the cellular location of TMPRSS2-activated SARS-CoV-2 entry favor fusion at the cell surface rather than from endosomal compartments because TMPRSS2 activity is $\mathrm{pH}$ independent. Ebola virus or SARS-CoV-2 accumulate in EEA1containing early endosomes $(3,20)$, a behavior consistent with the disruption of endolysosomal traffic that occurs when PIKfyve kinase activity is inhibited by genetic or pharmacological means $(21,40,42)$. It is therefore possible that changes in the endosomal milieu, combined with the redirection of SARS-CoV-2 and/or TMPRSS2 traffic in the presence of apilimod, might make infection more dependent on TMPRSS2 pharmacological inactivation than in its absence. The combined presence of apilimod and camostat or nafamostat mesylate would manifest as synergy of these compounds to prevent infection. These observations would also be consistent with the favored entry of TMPRSS2-activated SARS-CoV-2 from internal membrane compartments rather than from the cell surface.

The strong synergy that we describe in the laboratory setting for single cell types upon the combined use of the PIKfyve kinase and TMPRSS2 inhibitors highlights the potential clinical advantage of using a similar strategy to ameliorate the viral load and potentially reduce the risk of COVID-19 in patients infected with SARS-CoV-2 based on the simultaneous inhibition of these enzymes. Variants of apilimod and camostat, 
rendered more soluble by mesylate modification, are currently in clinical trials as orally administered inhibitors dispensed alone for various indications, including COVID-19 (7, $43,44)$. The more soluble inhibitor nafamostat mesylate has been administered intravenously to treat COVID-19 patients (8). We do not yet know how efficiently these inhibitors reach nasopharyngeal and lung tissues; nevertheless, we surmise that the combined use of these PIKfyve and TMPRSS2 inhibitors might also recapitulate the $\sim 5$ to 10 -fold increase in inhibitor efficiency that we observe in the laboratory setting, offering a considerable advantage for clinical application.

\section{MATERIALS AND METHODS}

Materials. The following reagents were purchased as indicated: Dulbecco modified Eagle's medium (DMEM) supplemented with $4.5 \mathrm{~g} /$ liter glucose, L-glutamine, and sodium pyruvate (catalog number 10013-CV; Corning, Inc.), fetal bovine serum (FBS) (catalog number S11150H; Atlanta Biologicals), a 100x solution of penicillin-streptomycin (catalog number 45000-652; VWR), camostat mesylate (catalog number SML0057; Sigma-Aldrich), E-64 (catalog number sc-201276; Santa Cruz Biotechnology), nafamostat mesylate (catalog number 14837; Cayman Chemical Company), apilimod (catalog number HY-14644; MedChem Express), dimethyl sulfoxide (DMSO) (catalog number 26855; Sigma-Aldrich), round cover glass number 1.5 (25 mm, catalog number C8-1.5H-N; Cellvis), polydimethylsiloxane (PDMS) (Sylgard, catalog number DC4019862; Krayden), isopropyl alcohol (catalog number 9080-03/MK303108; VWR), potassium hydroxide (catalog number 484016; Sigma-Aldrich), wheat germ agglutinin (WGA)-Alexa Fluor 647 (catalog number W32466; Invitrogen), paraformaldehyde (catalog number P6148; Sigma-Aldrich), dibasic sodium phosphate (catalog number BP329-1; Thermo Fisher Scientific), monobasic potassium phosphate (catalog number P5379; Sigma-Aldrich), sodium chloride (catalog number SX0420-5; EMD Millipore), potassium chloride (catalog number P217-500; Thermo Fisher Scientific), Tris (catalog number T-400-500; Goldbio), EDTA (catalog number E5134; Sigma-Aldrich), sucrose (catalog number S0389; Sigma-Aldrich), fetal calf serum (FCS) (catalog number SH30073.03; HyClone), bovine serum albumin (GE Healthcare), Triton X-100 (catalog number 28314; Thermo Fisher), Hoechst DNA stain (catalog number 62249; Thermo Fisher), L-glutamine (catalog number G7513; Sigma), Hoechst DNA dye (catalog number H6021; Sigma-Aldrich), and Alexa Fluor 647 fluorescently labeled goat anti-rabbit antibody (catalog number A32733; Thermo Fisher).

Purification of VSV-SARS-CoV-2 chimeras. The generation of recombinant VSV (Indiana serotype) expressing eGFP (VSV-eGFP) and VSV-eGFP chimeras whose glycoprotein G was replaced with either the wild-type spike (S) protein of the SARS-CoV-2 Wuhan-Hu-1 strain (VSV-SARS-CoV-2) or the point mutant D614G (VSV-SARS-CoV-2 D614G) was done as described previously (17). Briefly, VSV was grown by infection of BSR-T7/5 cells, and VSV-SARS-CoV-2 chimeras were grown by infection of MA104 cells. Cells were grown in 12 to 18 150-mm dishes and infected at a multiplicity of infection (MOI) of 0.01. The virus-containing supernatant was collected at $48 \mathrm{~h}$ postinfection. The supernatant was clarified by low-speed centrifugation at $1,000 \times g$ for $10 \mathrm{~min}$ at room temperature. An initial pellet of virus and extracellular particles was generated by centrifugation in a Ti45 fixed-angle rotor at 30,000 $\times g(25,000 \mathrm{rpm})$ for $2 \mathrm{~h}$ at $4^{\circ} \mathrm{C}$. The pellet was resuspended overnight in $1 \times \mathrm{NTE}(100 \mathrm{mM} \mathrm{NaCl}, 10 \mathrm{mM}$ Tris- $\mathrm{HCl}[\mathrm{pH} 7.4], 1 \mathrm{mM}$ EDTA) at $4^{\circ} \mathrm{C}$. The resuspended pellet was layered on top of a $15 \%$ sucrose-NTE cushion and subjected to ultracentrifugation in an SW55 swinging-bucket rotor at $110,000 \times g(35,000 \mathrm{rpm})$ for $2 \mathrm{~h}$ at $4^{\circ} \mathrm{C}$. The supernatant was aspirated, and the virus pellet was resuspended in $1 \times$ NTE overnight at $4^{\circ} \mathrm{C}$. The resuspended virus pellet was separated on a 15 to $45 \%$ sucrose-NTE gradient by ultracentrifugation in an SW55 swinging-bucket rotor at $150,000 \times g(40,000 \mathrm{rpm})$ for $1.5 \mathrm{~h}$ at $4^{\circ} \mathrm{C}$. The predominant lightscattering virus band was observed in the lower one-third of the gradient and was extracted by side puncture of the gradient tube. Extracted virus was diluted in $1 \times$ NTE and collected by ultracentrifugation in a Ti60 fixed-angle rotor at $115,000 \times g(40,000 \mathrm{rpm})$ for $2 \mathrm{~h}$ at $4^{\circ} \mathrm{C}$. The final pellet was resuspended overnight in $1 \times$ NTE in a volume of 0.2 to $0.5 \mathrm{ml}$ depending on the size of the pellet and stored at $4^{\circ} \mathrm{C}$ for use in subsequent imaging experiments.

Isolation and propagation of SARS-CoV-2. Human samples were obtained under Helsinki University Hospital laboratory research permit 30 HUS/32/2018\$16. For the isolation of SARS-CoV-2 from a COVID19 patient, briefly, a nasopharyngeal swab in $500 \mu$ l of Copan universal transport medium (UTM) was inoculated on Calu-3 cells (P1) and incubated for $1 \mathrm{~h}$ at $37^{\circ} \mathrm{C}$, after which the inoculum was removed and replaced with minimum essential medium supplemented with $2 \% \mathrm{FBS}$, L-glutamine, penicillin, and streptomycin. Virus replication was determined by real-time PCR (RT-PCR) for SARS-CoV-2 RNA-dependent RNA polymerase (RdRP) (45), and the infectious virus was collected $48 \mathrm{~h}$ after inoculation. The P1 stock was propagated once (P2) in VeroE6 + TMPRSS2 cells, sequenced, and stored at $-80^{\circ} \mathrm{C}$. Virus stocks were stored in a solution containing DMEM, 2\% FCS, 2 mM L-glutamine, and 1\% penicillin-streptomycin.

Cell culture. VeroE6 (ATCC CRL-1586), Caco-2 (ATCC HTB-37), and Calu-3 (ATCC HTB-55) cells were purchased from the ATCC. VeroE6+TMPRSS2 cells were a gift from Siyan Ding (14). VeroE6, VeroE6+TMPRSS2, Caco-2, and Calu-3 cells were maintained in DMEM supplemented with $25 \mathrm{mM}$ HEPES (pH 7.4), 10\% fetal bovine serum, and $1 \%$ penicillin-streptomycin. VeroE6 and VeroE6+TMPRSS2 cells were grown at $37^{\circ} \mathrm{C}$ with $5 \% \mathrm{CO}_{2}$ and split at a ratio of 1:10 every 3 to 4 days when cells were $\sim 90 \%$ confluent. Caco- 2 cells were grown at $37^{\circ} \mathrm{C}$ with $5 \% \mathrm{CO}_{2}$ and split at a ratio of $1: 5$ every 3 to 4 days when cells were $\sim 95 \%$ confluent. Calu-3 cells were grown at $37^{\circ} \mathrm{C}$ with $7 \% \mathrm{CO}_{2}$ and split at a ratio of $1: 3$ every 5 to 6 days when cells were $\sim 95 \%$ confluent. BSR-T5/T7 cells (46) were grown in DMEM supplemented with $10 \%$ FBS and $1 \%$ penicillin- 
streptomycin at $37^{\circ} \mathrm{C}$ with $5 \% \mathrm{CO}_{2}$ and split at a ratio of $1: 20$ every 2 to 3 days when cells were $\sim 90 \%$ confluent. MA104 cells (ATCC CRL-2378.1) were grown in medium 199 supplemented with 10\% FBS and 1\% penicillin-streptomycin. MA104 cells were grown at $37^{\circ} \mathrm{C}$ with $5 \% \mathrm{CO}_{2}$ and split at a ratio of $1: 3$ every 2 days when cells were $\sim 90 \%$ confluent. The medium was changed in all cell types every 2 days and regularly tested for the presence of mycoplasma.

Caco-2 cells stably expressing human ACE2 were generated by transduction with the third-generation lentivirus pLenti7.3/V5 DEST ACE2-EmGFP (prepared by the cloning facility Dream-Lab, Institute of Biotechnology, University of Helsinki, Helsinki, Finland); the expression of emerald GFP is driven by the simian virus 40 (SV40) promoter. Cells expressing eGFP were isolated with a fluorescence-activated cell sorter (FACS). These cells were grown in DMEM supplemented with $10 \%$ fetal calf serum, $1 \%$ penicillinstreptomycin, and $2 \mathrm{mM}$ L-glutamine. Cells were kept at $37^{\circ} \mathrm{C}$ with $5 \% \mathrm{CO}_{2}$ and split every 2 to 4 days when $\sim 90 \%$ confluent.

Infection protocol for VSV and VSV-SARS-CoV-2. Polydimethylsiloxane was cured by vigorous mixing with a curing reagent at a ratio of 1 to 10. PDMS was poured into a 10-cm petri dish (5 g of PDMS per plate) and incubated at $90^{\circ} \mathrm{C}$ for $\sim 4 \mathrm{~h}$. Plates were removed and stored at room temperature until use. PDMS was removed from the petri dish using a razor blade. Three-millimeter holes were punched into PDMS, which was cut with a blade to be $\sim 6$ to $10 \mathrm{~mm}$. Glass slides were cleaned by sonication first in isopropanol for $20 \mathrm{~min}$ and then in $0.5 \mathrm{M}$ potassium hydroxide for $20 \mathrm{~min}$, followed by extensive washing in MilliQ water. Glass was dried in an oven at $60^{\circ} \mathrm{C}$ for $30 \mathrm{~min}$ and then bonded to PDMS by exposing PDMS and glass to air plasma at 750 mtorr at $30 \mathrm{~W}$ for 2 min using a PDC-001 plasma cleaner (Harrick Plasma) and then firmly pressing the glass and PDMS together. This was followed by placing bonded glass and PDMS in an oven at $90^{\circ} \mathrm{C}$ for $20 \mathrm{~min}$. The glass coverslips mounted with a PDMS well were then placed in $70 \%$ ethanol for $10 \mathrm{~min}$ for sterilization prior to use for cell culture.

On the day prior to the experiment, cells were plated in PDMS wells on the glass slide stored in a 6well plate at a density to achieve $\sim 70 \%$ confluence on the day of the experiment. On the day of the experiment, cells were incubated with the desired inhibitor concentration for $1 \mathrm{~h}$. Medium was then removed, and virus that had been diluted into medium containing the indicated inhibitor concentration was added to the well in a volume of $10 \mu \mathrm{l}$. Medium was left in the 6-well plate outside the PDMS well at a level lower than the height of the PDMS well to maintain humidity and prevent evaporation. After the virus was incubated with the cells for $1 \mathrm{~h}$, the cells were washed with medium containing the indicated inhibitor, and the well was then filled with fresh medium. In all experiments, cells were kept at $37^{\circ} \mathrm{C}$ with $5 \%$ (for Vero, Vero+TMRPSS2, and Caco-2 cells) or $7 \%$ (for Calu-3 cells) $\mathrm{CO}_{2}$, and medium was prewarmed to $37^{\circ} \mathrm{C}$. Six hours after initiating the infection with VSV or $8 \mathrm{~h}$ after initiating the infection with VSV-SARS-CoV-2, medium was removed, and the cells were stained by adding $5 \mu \mathrm{g} / \mathrm{ml}$ WGA-Alexa Fluor 647 in phosphate-buffered saline (PBS) $\left(137 \mathrm{mM} \mathrm{NaCl}, 2.7 \mathrm{mM} \mathrm{KCl}, 8 \mathrm{mM} \mathrm{Na} \mathrm{HPO}_{4}, 2 \mathrm{mM} \mathrm{KH}_{2} \mathrm{PO}_{4}\right.$ [pH 7.4]) for $30 \mathrm{~s}$ at room temperature. Cells were then washed with sterile PBS 2 times and then fixed with 4\% paraformaldehyde in PBS. Infected cells were imaged using a spinning-disk confocal microscope with a $40 \times$ oil objective and a pixel size of $0.33 \mu \mathrm{m}$ where 20 optical planes were taken $0.5 \mu \mathrm{m}$ apart for every field of view (47). Cells were considered infected when they displayed a cytosolic eGFP fluorescence signal with a relative intensity 1.4 times that of the background of uninfected cells. Example images are maximum-intensity projections of the cell volume where the outline (white) line was obtained by tracing the WGA-Alexa Fluor 647 signal outlining the cell.

Infection protocol for SARS-CoV-2. All experiments with SARS-CoV-2 were performed in biosafety level 3 (BSL3) facilities at the University of Helsinki with appropriate institutional permits. Virus samples were obtained under Helsinki University Hospital laboratory research permit $30 \mathrm{HUS} / 32 / 2018 \S 16$. Virus titers were determined by a plaque assay in VeroE6+TMPRSS2 cells. Cells in DMEM, supplemented with 10\% FBS, 2 mM L-glutamine, 1\% penicillin-streptomycin, and $20 \mathrm{mM} \mathrm{HEPES} \mathrm{(pH} \mathrm{7.2),} \mathrm{were} \mathrm{seeded} 48 \mathrm{~h}$ before treatment at a density of 15,000 cells per well in 96-well imaging plates (catalog number 6005182; PerkinElmer). Inhibitors, or the DMSO control, were added either 60 min before infection or 90 min after infection at an $\mathrm{MOI}$ of $0.5 \mathrm{PFU}$ per cell. Infections were carried for $20 \mathrm{~h}$ at $37^{\circ} \mathrm{C}$ with $5 \% \mathrm{CO}_{2}$. Cells were then fixed with $4 \%$ paraformaldehyde in PBS for $30 \mathrm{~min}$ at room temperature before being processed for immunodetection of viral $\mathrm{N}$ protein, automated fluorescence imaging, and image analysis. Briefly, viral NP was detected with an in-house-developed rabbit polyclonal antibody (34) counterstained with Alexa Fluor 647-conjugated goat anti-rabbit secondary antibody, and nuclear staining was done using Hoechst DNA dye. Automated fluorescence imaging was done using a Molecular Devices ImageXpress Nano high-content epifluorescence microscope equipped with a $20 \times$ objective and a 4.7-megapixel CMOS (complementary metal oxide semiconductor) camera (pixel size, $0.332 \mu \mathrm{m}$ ). Image analysis was performed with CellProfiler-3 software (www.cellprofiler.org). Automated detection of nuclei was performed using the Otsu algorithm inbuilt in the software. To automatically identify infected cells, an area surrounding each nucleus (5-pixel expansion of the nuclear area) was used to estimate the fluorescence intensity of the viral NP immunolabeled protein, using an intensity threshold such that $<0.01 \%$ of positive cells were detected in noninfected wells.

Statistical analysis. The significance of the response (synergy $\delta$-score) upon the combined use of two drugs (Fig. 3) was calculated using the Bliss reference model for the combination of two drugs incorporated in the stand-alone Web application SynergyFinder v2 (23). This model assumes a stochastic process in which the effects of two drugs act independently. $\delta$-score values of between -10 and 10 suggest an additive interaction, and $\delta$-scores of $>10$ suggest that the interaction is synergistic.

The $\mathrm{EC}_{50}$ values in Fig. $2 \mathrm{~B}$ and $\mathrm{C}$ and Fig. 4 were obtained from replicate determinations calculated with least-squares nonlinear curve fitting using Igor Pro (WaveMetrics). An unpaired $t$ test was then used 
to determine the statistical significance in the difference in the $\mathrm{EC}_{50}$ values of camostat mesylate as a function of apilimod.

\section{ACKNOWLEDGMENTS}

We thank Sean Whelan and Judy White for comments and suggestions. We thank Tegy John Vadakkan for maintaining the spinning-disk confocal microscope.

A.J.B.K. carried out all the experiments in Fig. 1 to 3. R.O. and G.B. carried out the experiments in Fig. 4. A.S. maintained the cell lines and assisted with infectivity assays. R.O. generated Caco2-ACE2+EmGFP cells and propagated SARS-CoV-2 under the supervision of O.V. J.D.P. generated the stocks of VSV-SARS-CoV-2 chimeras. T.K. and A.J.B.K. were responsible for the overall design of the study. T.K. and A.J.B.K. drafted the manuscript. All authors commented on the manuscript.

This research was supported by NIH Maximizing Investigators' Research Award (MIRA) GM130386, research grants from the Danish Technical University and SANA, and unrestricted funds from IONIS to T.K.; a Harvard Virology Program NIH training grant T32 Al07245 postdoctoral fellowship to A.J.B.K.; Academy of Finland research grant 336490, the Jane and Aatos Erkko Foundation, the EU Horizon 2020 program VEO 874735, and Helsinki University Hospital fund TYH2018322 to O.V.; Academy of Finland research grant 318434 and unrestricted funds supporting COVID-19 research to G.B.; and funds from the University of Helsinki Graduate Program in Microbiology and Biotechnology to R.O.

T.K. is a member of the Medical Advisory Board of Al Therapeutics, Inc.

\section{REFERENCES}

1. Hoffmann M, Kleine-Weber H, Schroeder S, Kruger N, Herrler T, Erichsen $\mathrm{S}$, Schiergens TS, Herrler G, Wu NH, Nitsche A, Muller MA, Drosten C, Pohlmann S. 2020. SARS-CoV-2 cell entry depends on ACE2 and TMPRSS2 and is blocked by a clinically proven protease inhibitor. Cell 181: 271-280.e8. https://doi.org/10.1016/j.cell.2020.02.052.

2. Hoffmann M, Schroeder S, Kleine-Weber H, Muller MA, Drosten C, Pohlmann S. 2020. Nafamostat mesylate blocks activation of SARS-CoV-2: new treatment option for COVID-19. Antimicrob Agents Chemother 64: e00754-20. https://doi.org/10.1128/AAC.00754-20.

3. Kang YL, Chou YY, Rothlauf PW, Liu Z, Soh TK, Cureton D, Case JB, Chen RE, Diamond MS, Whelan SPJ, Kirchhausen T. 2020. Inhibition of PIKfyve kinase prevents infection by Zaire ebolavirus and SARS-CoV-2. Proc Natl Acad Sci U S A 117:20803-20813. https://doi.org/10.1073/pnas.2007837117.

4. Ou X, Liu Y, Lei X, Li P, Mi D, Ren L, Guo L, Guo R, Chen T, Hu J, Xiang Z, Mu Z, Chen X, Chen J, Hu K, Jin Q, Wang J, Qian Z. 2020. Characterization of spike glycoprotein of SARS-CoV-2 on virus entry and its immune crossreactivity with SARS-CoV. Nat Commun 11:1620. https://doi.org/10.1038/ s41467-020-15562-9.

5. Riva L, Yuan S, Yin X, Martin-Sancho L, Matsunaga N, Pache L, BurgstallerMuehlbacher S, De Jesus PD, Teriete P, Hull MV, Chang MW, Chan JF, Cao J, Poon VK, Herbert KM, Cheng K, Nguyen TH, Rubanov A, Pu Y, Nguyen C, Choi A, Rathnasinghe R, Schotsaert M, Miorin L, Dejosez M, Zwaka TP, Sit KY, Martinez-Sobrido L, Liu WC, White KM, Chapman ME, Lendy EK, Glynne RJ, Albrecht R, Ruppin E, Mesecar AD, Johnson JR, Benner C, Sun R, Schultz PG, Su AI, Garcia-Sastre A, Chatterjee AK, Yuen KY, Chanda SK. 2020. Discovery of SARS-CoV-2 antiviral drugs through large-scale compound repurposing. Nature 586:113-119. https://doi.org/10.1038/s41586 -020-2577-1

6. Yamamoto $M$, Kiso $M$, Sakai-Tagawa $Y$, Iwatsuki-Horimoto $K$, Imai $M$, Takeda M, Kinoshita N, Ohmagari N, Gohda J, Semba K, Matsuda Z, Kawaguchi Y, Kawaoka Y, Inoue Jl. 2020. The anticoagulant nafamostat potently inhibits SARS-CoV-2 S protein-mediated fusion in a cell fusion assay system and viral infection in vitro in a cell-type-dependent manner. Viruses 12:629. https://doi.org/10.3390/v12060629.

7. Gunst JD, Staerke NB, Pahus MH, Kristensen LH, Bodilsen J, Lohse N, Dalgaard LS, Brønnum D, Fröbert O, Hønge B, Johansen IS, Monrad I, Erikstrup C, Rosendal R, Vilstrup E, Mariager T, Bove DG, Offersen R, Shakar S, Cajander S, Jørgensen NP, Sritharan SS, Breining P, Jespersen S, Mortensen $\mathrm{KL}$, Jensen $\mathrm{ML}$, Kolte L, Frattari GS, Larsen $\mathrm{CS}$, Storgaard $\mathrm{M}$, Nielsen LP, Tolstrup M, Sædder EA, Østergaard LJ, Ngo HTT, Jensen MH, Højen JF, Kjolby M, Søgaard OS. 2021. Efficacy of the TMPRSS2 inhibitor camostat mesilate in patients hospitalized with Covid-19-a double-blind randomized controlled trial. EClinicalMedicine 35:100849. https://doi.org/ 10.1016/j.eclinm.2021.100849.

8. Doi K, Ikeda M, Hayase N, Moriya K, Morimura N, COVID-UTH Study Group. 2020. Nafamostat mesylate treatment in combination with favipiravir for patients critically ill with Covid-19: a case series. Crit Care 24:392. https:// doi.org/10.1186/s13054-020-03078-z.

9. Huang Y, Yang C, Xu XF, Xu W, Liu SW. 2020. Structural and functional properties of SARS-CoV-2 spike protein: potential antivirus drug development for COVID-19. Acta Pharmacol Sin 41:1141-1149. https://doi.org/10 .1038/s41401-020-0485-4.

10. Shang J, Wan Y, Luo C, Ye G, Geng Q, Auerbach A, Li F. 2020. Cell entry mechanisms of SARS-CoV-2. Proc Natl Acad Sci U S A 117:11727-11734. https://doi.org/10.1073/pnas.2003138117.

11. Wrapp D, Wang N, Corbett KS, Goldsmith JA, Hsieh CL, Abiona O, Graham BS, McLellan JS. 2020. Cryo-EM structure of the 2019-nCoV spike in the prefusion conformation. Science 367:1260-1263. https://doi.org/10.1126/ science.abb2507.

12. Yan R, Zhang Y, Li Y, Xia L, Guo Y, Zhou Q. 2020. Structural basis for the recognition of SARS-CoV-2 by full-length human ACE2. Science 367: 1444-1448. https://doi.org/10.1126/science.abb2762.

13. Yang J, Petitjean SJL, Koehler M, Zhang Q, Dumitru AC, Chen W, Derclaye S, Vincent SP, Soumillion P, Alsteens D. 2020. Molecular interaction and inhibition of SARS-CoV-2 binding to the ACE2 receptor. Nat Commun 11: 4541. https://doi.org/10.1038/s41467-020-18319-6.

14. Zang R, Gomez Castro MF, McCune BT, Zeng Q, Rothlauf PW, Sonnek NM, Liu Z, Brulois KF, Wang X, Greenberg HB, Diamond MS, Ciorba MA, Whelan SPJ, Ding S. 2020. TMPRSS2 and TMPRSS4 promote SARS-CoV-2 infection of human small intestinal enterocytes. Sci Immunol 5:eabc3582. https://doi.org/10.1126/sciimmunol.abc3582.

15. Du L, Kao RY, Zhou Y, He Y, Zhao G, Wong C, Jiang S, Yuen KY, Jin DY, Zheng BJ. 2007. Cleavage of spike protein of SARS coronavirus by protease factor $\mathrm{Xa}$ is associated with viral infectivity. Biochem Biophys Res Commun 359:174-179. https://doi.org/10.1016/j.bbrc.2007.05.092.

16. Frydman GH, Streiff MB, Connors JM, Piazza G. 2020. The potential role of coagulation factor Xa in the pathophysiology of COVID-19: a role for anticoagulants as multimodal therapeutic agents. TH Open 4:e288-e299. https://doi.org/10.1055/s-0040-1718415.

17. Case JB, Rothlauf PW, Chen RE, Liu Z, Zhao H, Kim AS, Bloyet L-M, Zeng Q, Tahan S, Droit L, Ilagan MXG, Tartell MA, Amarasinghe G, Henderson JP, Miersch S, Ustav M, Sidhu S, Virgin HW, Wang D, Ding S, Corti D, Theel ES, Fremont DH, Diamond MS, Whelan SPJ. 2020. Neutralizing antibody and soluble ACE2 inhibition of a replication-competent VSV-SARS-CoV-2 and 
a clinical isolate of SARS-CoV-2. Cell Host Microbe 28:475-485.e5. https:// doi.org/10.1016/j.chom.2020.06.021.

18. Gonzalez-Hernandez M, Muller A, Hoenen T, Hoffmann M, Pohlmann S. 2019. Calu-3 cells are largely resistant to entry driven by filovirus glycoproteins and the entry defect can be rescued by directed expression of DC-SIGN or cathepsin L. Virology 532:22-29. https://doi.org/10.1016/j virol.2019.03.020.

19. Dittmar M, Lee JS, Whig K, Segrist E, Li M, Kamalia B, Castellana L, Ayyanathan K, Cardenas-Diaz FL, Morrisey EE, Truitt R, Yang W, Jurado K, Samby K, Ramage H, Schultz DC, Cherry S. 2021. Drug repurposing screens reveal cell-type-specific entry pathways and FDA-approved drugs active against SARS-Cov-2. Cell Rep 35:108959. https://doi.org/10.1016/j .celrep.2021.108959.

20. Nelson EA, Dyall J, Hoenen T, Barnes AB, Zhou H, Liang JY, Michelotti J, Dewey WH, DeWald LE, Bennett RS, Morris PJ, Guha R, Klumpp-Thomas C, McKnight C, Chen YC, Xu X, Wang A, Hughes E, Martin S, Thomas C, Jahrling PB, Hensley LE, Olinger GG, Jr, White JM. 2017. The phosphatidylinositol-3-phosphate 5-kinase inhibitor apilimod blocks filoviral entry and infection. PLoS Negl Trop Dis 11:e0005540. https://doi.org/10.1371/journal .pntd.0005540.

21. Baranov MV, Bianchi F, Schirmacher A, van Aart MAC, Maassen S, Muntjewerff EM, Dingjan I, Ter Beest M, Verdoes M, Keyser SGL, Bertozzi CR, Diederichsen U, van den Bogaart G. 2019. The phosphoinositide kinase PIKfyve promotes cathepsin-S-mediated major histocompatibility complex class II antigen presentation. iScience 11:160-177. https://doi .org/10.1016/j.isci.2018.12.015.

22. Gayle S, Landrette S, Beeharry N, Conrad C, Hernandez M, Beckett P, Ferguson SM, Mandelkern T, Zheng M, Xu T, Rothberg J, Lichenstein $\mathrm{H}$. 2017. Identification of apilimod as a first-in-class PIKfyve kinase inhibitor for treatment of B-cell non-Hodgkin lymphoma. Blood 129:1768-1778. https://doi.org/10.1182/blood-2016-09-736892.

23. lanevski A, Giri AK, Aittokallio T. 2020. SynergyFinder 2.0: visual analytics of multi-drug combination synergies. Nucleic Acids Res 48:W488-W493. https://doi.org/10.1093/nar/gkaa216.

24. Bliss C. 1939. The toxicity of poisons applied jointly. Ann Appl Biol 26: 585-615. https://doi.org/10.1111/j.1744-7348.1939.tb06990.x.

25. Padmanabhan P, Desikan R, Dixit NM. 2020. Targeting TMPRSS2 and cathepsin B/L together may be synergistic against SARS-CoV-2 infection. PLoS Comput Biol 16:e1008461. https://doi.org/10.1371/journal.pcbi.1008461.

26. Park JE, Li K, Barlan A, Fehr AR, Perlman S, McCray PB, Jr, Gallagher T. 2016. Proteolytic processing of Middle East respiratory syndrome coronavirus spikes expands virus tropism. Proc Natl Acad Sci U S A 113: 12262-12267. https://doi.org/10.1073/pnas.1608147113.

27. Murgolo N, Therien AG, Howell B, Klein D, Koeplinger K, Lieberman LA, Adam GC, Flynn J, McKenna P, Swaminathan G, Hazuda DJ, Olsen DB. 2021. SARS-CoV-2 tropism, entry, replication, and propagation: considerations for drug discovery and development. PLoS Pathog 17:e1009225. https://doi.org/10.1371/journal.ppat.1009225.

28. Mykytyn AZ, Breugem TI, Riesebosch S, Schipper D, van den Doel PB, Rottier RJ, Lamers MM, Haagmans BL. 2021. SARS-CoV-2 entry into human airway organoids is serine protease-mediated and facilitated by the multibasic cleavage site. Elife 10:e64508. https://doi.org/10.7554/eLife.64508.

29. Koch J, Uckeley ZM, Doldan P, Stanifer M, Boulant S, Lozach P-Y. 2020. Host cell proteases drive early or late SARS-CoV-2 penetration. bioRxiv https://doi.org/10.1101/2020.12.22.423906.

30. Plante JA, Liu Y, Liu J, Xia H, Johnson BA, Lokugamage KG, Zhang X, Muruato AE, Zou J, Fontes-Garfias CR, Mirchandani D, Scharton D, Bilello JP, Ku Z, An Z, Kalveram B, Freiberg AN, Menachery VD, Xie X, Plante KS, Weaver SC, Shi PY. 2021. Spike mutation D614G alters SARS-CoV-2 fitness. Nature 592:116-121. https://doi.org/10.1038/s41586-020-2895-3.

31. Gobeil SM, Janowska K, McDowell S, Mansouri K, Parks R, Manne K, Stalls V, Kopp MF, Henderson R, Edwards RJ, Haynes BF, Acharya P. 2021. D614G mutation alters SARS-CoV-2 spike conformation and enhances protease cleavage at the S1/S2 junction. Cell Rep 34:108630. https://doi .org/10.1016/j.celrep.2020.108630.

32. Yurkovetskiy L, Wang $X$, Pascal KE, Tomkins-Tinch $C$, Nyalile TP, Wang $Y$, Baum A, Diehl WE, Dauphin A, Carbone C, Veinotte K, Egri SB, Schaffner SF, Lemieux JE, Munro JB, Rafique A, Barve A, Sabeti PC, Kyratsous CA, Dudkina NV, Shen K, Luban J. 2020. Structural and functional analysis of the D614G SARS-CoV-2 spike protein variant. Cell 183:739-751.e8. https://doi.org/10 .1016/j.cell.2020.09.032.
33. Zhang $L$, Jackson $C B$, Mou $H$, Ojha A, Peng $H$, Quinlan BD, Rangarajan ES, Pan A, Vanderheiden A, Suthar MS, Li W, Izard T, Rader C, Farzan M, Choe H. 2020. SARS-CoV-2 spike-protein D614G mutation increases virion spike density and infectivity. Nat Commun 11:6013. https://doi.org/10.1038/ s41467-020-19808-4.

34. Cantuti-Castelvetri L, Ojha R, Pedro LD, Djannatian M, Franz J, Kuivanen S, van der Meer F, Kallio K, Kaya T, Anastasina M, Smura T, Levanov L, Szirovicza L, Tobi A, Kallio-Kokko H, Osterlund P, Joensuu M, Meunier FA, Butcher SJ, Winkler MS, Mollenhauer B, Helenius A, Gokce O, Teesalu T, Hepojoki J, Vapalahti O, Stadelmann C, Balistreri G, Simons M. 2020. Neuropilin-1 facilitates SARS-CoV-2 cell entry and infectivity. Science 370:856-860. https://doi.org/10.1126/science.abd2985.

35. Bayati A, Kumar R, Francis V, McPherson PS. 2021. SARS-CoV-2 infects cells following viral entry via clathrin-mediated endocytosis. J Biol Chem 296: 100306. https://doi.org/10.1016/j.jbc.2021.100306.

36. Khan N, Chen X, Geiger JD. 2020. Role of endolysosomes in severe acute respiratory syndrome coronavirus- 2 infection and coronavirus disease 2019 pathogenesis: implications for potential treatments. Front Pharmacol 11:595888. https://doi.org/10.3389/fphar.2020.595888.

37. Uhlen M, Fagerberg L, Hallstrom BM, Lindskog C, Oksvold P, Mardinoglu A, Sivertsson A, Kampf C, Sjostedt E, Asplund A, Olsson I, Edlund K, Lundberg E, Navani S, Szigyarto CA-K, Odeberg J, Djureinovic D, Takanen JO, Hober S, Alm T, Edqvist P-H, Berling H, Tegel H, Mulder J, Rockberg J, Nilsson P, Schwenk JM, Hamsten M, von Feilitzen K, Forsberg M, Persson L, Johansson F, Zwahlen M, von Heijne G, Nielsen J, Ponten F. 2015. Proteomics. Tissuebased map of the human proteome. Science 347:1260419. https://doi.org/ $10.1126 /$ science. 1260419.

38. Glowacka I, Bertram S, Muller MA, Allen P, Soilleux E, Pfefferle S, Steffen I, Tsegaye TS, He Y, Gnirss K, Niemeyer D, Schneider H, Drosten C, Pohlmann S. 2011. Evidence that TMPRSS2 activates the severe acute respiratory syndrome coronavirus spike protein for membrane fusion and reduces viral control by the humoral immune response. J Virol 85:4122-4134. https://doi .org/10.1128/JVI.02232-10.

39. de Lartigue J, Polson H, Feldman M, Shokat K, Tooze SA, Urbe S, Clague MJ. 2009. PIKfyve regulation of endosome-linked pathways. Traffic 10: 883-893. https://doi.org/10.1111/j.1600-0854.2009.00915.x.

40. Kim GH, Dayam RM, Prashar A, Terebiznik M, Botelho RJ. 2014. PIKfyve inhibition interferes with phagosome and endosome maturation in macrophages. Traffic 15:1143-1163. https://doi.org/10.1111/tra.12199.

41. Dyall J, Nelson EA, DeWald LE, Guha R, Hart BJ, Zhou H, Postnikova E, Logue J, Vargas WM, Gross R, Michelotti J, Deiuliis N, Bennett RS, Crozier I, Holbrook MR, Morris PJ, Klumpp-Thomas C, McKnight C, Mierzwa T, Shinn P, Glass PJ, Johansen LM, Jahrling PB, Hensley LE, Olinger GG, Jr, Thomas C, White JM. 2018. Identification of combinations of approved drugs with synergistic activity against Ebola virus in cell cultures. J Infect Dis 218: S672-S678. https://doi.org/10.1093/infdis/jiy304.

42. Soares AC, Ferreira A, Marien J, Delay C, Lee E, Trojanowski JQ, Moechars D, Annaert W, De Muynck L. 2021. PIKfyve activity is required for lysosomal trafficking of tau aggregates and tau seeding. J Biol Chem 296: 100636. https://doi.org/10.1016/j.jbc.2021.100636.

43. Billich A. 2007. Drug evaluation: apilimod, an oral IL-12/IL-23 inhibitor for the treatment of autoimmune diseases and common variable immunodeficiency. IDrugs 10:53-59.

44. Uno Y. 2020. Camostat mesilate therapy for COVID-19. Intern Emerg Med 15:1577-1578. https://doi.org/10.1007/s11739-020-02345-9.

45. Corman VM, Landt O, Kaiser M, Molenkamp R, Meijer A, Chu DKW, Bleicker T, Brunink S, Schneider J, Schmidt ML, Mulders DGJC, Haagmans $B L$, van der Veer B, van den Brink S, Wijsman L, Goderski G, Romette J-L, Ellis J, Zambon M, Peiris M, Goossens H, Reusken C, Koopmans MPG, Drosten C. 2020. Detection of 2019 novel coronavirus (2019-nCoV) by real-time RT-PCR. Euro Surveill 25:2000045. https://doi.org/10.2807/1560 -7917.ES.2020.25.3.2000045.

46. Buchholz UJ, Finke S, Conzelmann KK. 1999. Generation of bovine respiratory syncytial virus (BRSV) from cDNA: BRSV NS2 is not essential for virus replication in tissue culture, and the human RSV leader region acts as a functional BRSV genome promoter. J Virol 73:251-259. https://doi.org/10 .1128/JVI.73.1.251-259.1999.

47. Cocucci E, Aguet F, Boulant S, Kirchhausen T. 2012. The first five seconds in the life of a clathrin-coated pit. Cell 150:495-507. https://doi.org/10 .1016/j.cell.2012.05.047. 